\title{
EFFETS DE LA TERRAMYCINE SUR LA PRODUCTION LAITIERE CHEZ LA VACHE ET SUR LA MATURATION DU FROMAGE
}

\author{
par
}

\author{
ALFIO FALASCHINI et PAOLO SOCINI
}

Université de Bologne, Institut de Zootechnie

Tous les Auteurs pensent que l'adjonction d'antibiotique à la ration alimentaire favorise la croissance des jeunes animaux, y compris les agneaux et les veaux de lait. La littérature sur les effets des antibiotiques sur la production du lait et du fromage, au contraire, n'est point unanime.

Dans la Revue de l'élevage de 1957 [1], on rapporte que la présence d'antibiotiques dans le lait a une remarquable répercussion sur l'industrie laitière, mais que par ailleurs il en faut d'importantes quantités pour empêcher la maturation de la crème, tandis que des antibiotiques seraient nuisibles pour celle du fromage. Les antibiotiques auraient un effet désastreux dans la fabrication du camembert par exemple.

KrIEnKe [2] aurait vu que le traitement des mastites avec la pénicilline (100.000 unités par quartier) causerait pendant plusieurs jours, la présence dans le lait de quantités d'antibiotique suffisantes pour retarder la production d'acide lactique. Le maximum de concentration apte à empêcher le commencement de la fermentation, d'après les auteurs serait de 0,1 unité par millilitre de lait : des quantités supérieures en effet causeraient une coagulation impropre du lait employé pour la fabrication du fromage.

ARNAUdi [3] rapporte que, selon les résultats de quelques recherches exécutées à l'Institut supérieur d'Agriculture danois, la présence de $0 \mathrm{mg} .02$ d'auréomycine ou de $0 \mathrm{mg} .20$ de chloromycine ou de $0 \mathrm{mg}$. 04 de streptomycine ou bien de $0 \mathrm{mgr}$. $01 \mathrm{de}$ terramycine, est suffisante pour inhiber le commencement de la multiplication des lactobacilles joints à un lait stérile et que l'auréomycine et la terramycine déploient, à l'égard des bactéries homofermentatives, une activité plus marquée qu'à l'égard-des hétérofermentatives.

Hunter [4], d'ailleurs, aurait vu que la sensibilité des diverses souches de bactéries envers les antibiotiques n'est pas la même. En effet, tandis que le développement du Streptococcus cremoris serait considérablement inhibé par une concentration de 0,1 unité de penicilline par millilitre, la concentration, pour obtenir le même effet sur le streptococcus lactis, doit être de 0,23-0,3 par millilitre.

Hargrove, Walter, Malkames et Maskell [5] constatèrent 
que, à la suite du traitement des mastites par la pénicilline, on a encore dans le lait de la deuxième traite $0,04 \%$ de l'antibiotique injecté; aucune trace, au contraire, ne fut mise par eux en évidence lorsqu'on employa la streptomycine. Les mêmes Auteurs virent en outre que la multiplication de lactobacillus bulgaricus du fromage suisse est inhibée seulement quand la concentration de la pénicilline est de 0,1 unité par millilitre de lait.

Wilkowske et KRIEnke [6] n'auraient pas remarqué des degrés divers de sensibilité envers la pénicilline de la part de trois variétés de lactobacille.

Les mêmes Auteurs [7], expérimentant sur divers types de fromage, auraient vu que l'adjonction d'antibiotiques ne modifie pas la marche de la maturation, si la concentration est inférieure à deux unités par millilitre de lait.

WARNER [8] LOOSLI et WARNER [9], après une administration de 700 milligrammes d'auréomycine chaque jour pendant six semaines, à des vaches en lactation, non seulement ne remarquèrent aucun effet nuisible sur l'activité des enzymes ajoutés au lait pour la fabrication des fromages, de façon que leur maturation resta parfaitement normale, mais ils ne réussirent pas non plus à mettre en évidence la moindre trace d'antibiotiques dans le lait obtenu des vaches ainsi traitées.

Ils n'auraient remarqué aucun effet significatif sur la production du lait à la suite de l'administration quotidienne de 100 milligrammes d'auréomycine.

Des résultats presque semblables furent obtenus par BARTLEY et Coll. [10] ; en effet, aucune variation de quantité et de qualité sur la production du lait ne fut remarquée par eux lorsqu' «ils administrèrent à des vaches en lactation $32 \mathrm{mg}$ d'auréomycine par jour et par 100 livres de poids vif.

Martin, Claydon et BARTLEy [11] traitant chaque jour quelques vaches en lactation avec 200-300 milligrammes d'auréomycine, non seulement ne remarquèrent pas d'effets nuisibles sur le nombre des germes du lait et sur son acidité, mais ils virent aussi que le Cheddar, que l'on fabriquait avec ce lait gardait entièrement ses qualités.

Des effets significatifs sur la production du lait au moyen de l'administration d'antibiotiques (bacitracine, auréomycine, auréofac, streptomycine, néomycine) ne furent pas constatés par HuFFMAN [12] non plus.

HAQ et al. [13], HAQ, Rusoff et Gelpi [14] Rusoff et HAQ [15] malgré l'administration de quantités élevées d'auréomycine, n'auraient pas constaté de variation significative sur la quantité et la qualité du lait, dont le contenu bactérien serait resté inchangé. 
Les Auteurs en outre n'auraient pas réussi à mettre en évidence des quantités dosables d'antibiotiques dans le lait des vaches traitées avec des antibiotiques.

\section{***}

Partant de l'avis que l'on a pu attribuer les résultats discordants à l'importante quantité d'antibiotiques, administrée par presque tous les expérimentateurs, par voie orale aussi, nous avons institué trois expériences, pour constater les effets, sur la production du lait et du fromage, de l'administration par voie orale, de petites quantités de terramycine.

\section{Première expérience}

Matériel et technique. - Les recherches ont été conduites, pendant trois périodes, sur neuf vaches du même âge et de la même race (Brune alpine), de la même période de lactation et du même nombre de parts.

De celles-ci, trois servirent de témoin et six reçurent, pendant la deuxième période expérimentale $1 \mathrm{gr}$. 5 de TM5, pro capite et pro die, (le TM5, comme on sait, est préparé par la Pfeizer Corporation et contient 11 grammes de terramycine par kilo de substance).

Les animaux furent tenus dans les mêmes conditions d'ambiance et d'alimentation : sur chaque sujet fut contrôlée à chaque traite, et pendant toute la durée de l'expérience, la production du lait et, dans un échantillon pris du lait des deux traites de la même journée, on détermina hebdomadairement le contenu en matière grasse et en protéines totales; on a élaboré les valeurs suivant la méthode de BRANDT, puis on les a soumises à l'analyse statistique.

Résultats. - Les valeurs moyennes sont rapportées dans les trois tableaux ci-après.

Considérant les valeurs contenues dans le tableau, il résulte que, tandis que dans les vaches témoins la quantité du lait penche vers la diminution, dans celles qui recevaient le TM5, au contraire, cette quantité est plus grande soit que celle de la première période, soit que celle de la troisième période. L'analyse statistique démontre que les valeurs sont significatives.

Considérant ces données, on voit que le contenu en matière grasse du lait des vaches des deux groupes penche vers la diminution très probablement à cause du régime vert auquel on a soumis tous les animaux vingt jours après le début de l'expérience ; le phénomène cependant est plus accentué chez les vaches qui, pendant la deuxième période, reçurent le TM5. La diminution, en comparaison avec celle des vaches témoins n'est pas, au point de vue statistique, significative. 


\section{TABLEAU I}

GROUPE DES VACHES AUXQUELLES ON A ADMINISTRE TM5: EFFETS SUR LA PRODUGTION DU LAIT

\begin{tabular}{|c|c|c|c|c|}
\hline Nom de la vache & $\begin{array}{l}\text { Ire période } \\
\mathrm{Kg} .\end{array}$ & $\begin{array}{l}\mathrm{II}^{\mathbf{e}} \text { période } \\
\mathrm{Kg} .\end{array}$ & $\begin{array}{l}\mathrm{III}^{\mathrm{e}} \text { pé- } \\
\text { riode } \mathrm{Kg} \text {. }\end{array}$ & $\begin{array}{c}\text { Différence } \\
\mathrm{I}-(2.11)+111\end{array}$ \\
\hline Aaltie $14 \ldots$ & 22,88 & 25,17 & 23,34 & $-4,12$ \\
\hline Bertha $\ldots \ldots \ldots \ldots \ldots$ & 15,63 & 18,02 & 15,58 & $-4,81$ \\
\hline Hildegonda $11 \quad \ldots \ldots \ldots$ & 15,24 & 17,03 & 15,72 & $-\quad 3,10$ \\
\hline Adele $3 \ldots \ldots \ldots$ & 12,06 & 13,53 & 11,26 & $-4,74$ \\
\hline Aspra $\ldots \ldots \ldots$ & 10,98 & 12,04 & 10,73 & $-\quad 2,37$ \\
\hline Stinjntie $\ldots \ldots \ldots \ldots$ & 6,85 & 8,09 & 6,76 & $-2,57$ \\
\hline Total & 83,64 & 93,88 & 83,39 & $-21,71$ \\
\hline Moyenne : vache par jour & 13,94 & 15,65 & 13,89 & $-3,62$ \\
\hline $\mathrm{E}=2,294$ & \multicolumn{4}{|c|}{$\mathrm{Ed}=2,4852$} \\
\hline
\end{tabular}

Groupe des vaches de contrôle : variation de la production du lait

\begin{tabular}{|c|c|c|c|c|}
\hline Neve $\ldots \ldots \ldots \ldots \ldots$ & 20,22 & 19,61 & 19,40 & $-0,40$ \\
\hline 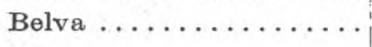 & 14,22 & 13,03 & 13,24 & $+1,40$ \\
\hline $\operatorname{Rea} \ldots \ldots \ldots \ldots \ldots$ & 11,58 & 11,02 & 10,51 & $+0,50$ \\
\hline Total $\ldots \ldots \ldots \ldots$ & 46,02 & 43,66 & 43,15 & $+1,50$ \\
\hline Moyenne : vache par jour & 15,94 & 14,55 & 14,38 & $+0,50$ \\
\hline $\mathbf{E}=0,950$ & $\mathrm{Dm}=$ & & 3,26 & $\mathrm{P}>0,01<0,02$ \\
\hline
\end{tabular}

TABLEAU II

GROUPE DES VAGHES AUXQUELLES ON A ADMINISTRE TM5: EFFETS SUR LE GONTENU EN MATIËRE GRASSE DU LAIT

\begin{tabular}{|c|c|c|c|c|}
\hline Nom de la vache & $\begin{array}{c}\text { Ire période } \\
\% \\
\end{array}$ & $\begin{array}{c}1 I^{\mathrm{e}} \text { période } \\
\%\end{array}$ & $\begin{array}{l}\text { IIII pé- } \\
\text { riode } \%\end{array}$ & $\begin{array}{c}\text { Différence } \\
1-(2.11)+111\end{array}$ \\
\hline Aaltie $14 \ldots \ldots \ldots \ldots$ & 3,40 & 2,32 & 3,21 & $-1,97$ \\
\hline Bertha $34 \ldots \ldots \ldots \ldots$ & 3,05 & 2,85 & 2,96 & $-0,31$ \\
\hline Hildegonda ........... & 3,39 & 2,80 & 3,13 & $-0,92$ \\
\hline Adele ........... & 3,26 & 3,52 & 3,61 & $+0,17$ \\
\hline Aspra $\ldots \ldots \ldots \ldots \ldots$ & 3,57 & 3,30 & 3,50 & $-0,47$ \\
\hline Stijntio $\quad \ldots \ldots \ldots \ldots \ldots$ & 4,55 & 4,50 & 4,56 & $-0,11$ \\
\hline Total $\ldots \ldots \ldots \ldots$ & 21,22 & 19,29 & 20,97 & $-3,61$ \\
\hline $\begin{array}{l}\text { Moyenne : vache par jour } \\
\mathrm{E}=0,3116\end{array}$ & 3,53 & 3,21 & 3,48 & $-0,60$ \\
\hline
\end{tabular}


TABLEAU II (suite)

GROUPE DES VAGHES TÉMOIN :

VARIATION DE LA PRODUGTION DE LA MATIÈRE GRASSE DU LAIT

\begin{tabular}{|c|c|c|c|c|}
\hline Nom de la vache & $\mid \begin{array}{c}\text { Ire période } \\
\%\end{array}$ & $\mid \begin{array}{c}\mathrm{II}^{\mathrm{e}} \text { période } \\
\%\end{array}$ & $\begin{array}{l}\text { III e pé- } \\
\text { riode } \%\end{array}$ & $\begin{array}{c}\text { Différence } \\
1-(2.11)+111\end{array}$ \\
\hline Neve $\ldots . . .$. & 4,40 & 4,02 & 4,05 & $-0,41$ \\
\hline Belva ............... & 4,36 & 4,25 & 4,10 & $+0,04$ \\
\hline Rea $\ldots \ldots \ldots \ldots \ldots \ldots$ & 4,42 & 4,32 & 4,35 & $-0,13$ \\
\hline Total $\ldots \ldots \ldots \ldots$ & 13,18 & 12,59 & 12,50 & $-0,50$ \\
\hline Moyenne : vache par jour & 4,29 & 4,19 & 4,16 & $-0,17$ \\
\hline$E=0,2374$ & $\begin{aligned} \mathrm{Dm} & = \\
\mathrm{P} & >\end{aligned}$ & $\begin{array}{l}0,43 \quad \mathrm{Ed} \\
0,3<0,04\end{array}$ & $=0,3994$ & $t=1,098$ \\
\hline
\end{tabular}

TABLEAU III

GROUPE DES VACHES AUXQUELLES ON A ADMINISTRE TM5: EFFETS SUR LE GONTENU EN PROTÉINES DU LAIT

\begin{tabular}{|c|c|c|c|c|}
\hline Nom de la vache & $\begin{array}{c}\text { Ire période } \\
\%\end{array}$ & $\mid \begin{array}{c}\text { II } \text { période } \\
\%\end{array}$ & $\begin{array}{l}\text { III e pé- } \\
\text { riode } \%\end{array}$ & $\begin{array}{c}\text { Différence } \\
1-(2.11)+111\end{array}$ \\
\hline Aaltie $14 \ldots \ldots \ldots \ldots$ & 2,61 & 2,91 & 2,72 & 0,49 \\
\hline Bertha $34 \ldots \ldots \ldots \ldots$ & 2,74 & 2,86 & 2,76 & 0,22 \\
\hline Hildegonda $11 \quad \ldots \ldots \ldots$ & 2,94 & 3,15 & 2,94 & 0,47 \\
\hline Adele $3 \quad \ldots \ldots \ldots \ldots$ & 3,01 & 3,17 & 3,02 & 0,31 \\
\hline Aspra $\ldots \ldots \ldots \ldots \ldots$ & 3 & 3,20 & 3,04 & 0,36 \\
\hline Stjintie $\ldots \ldots \ldots \ldots \ldots$ & 3,45 & 3,83 & 3,45 & 0,76 \\
\hline$\cdots \cdots \cdots \cdots$ & 17,75 & 19,12 & 17,93 & 2,61 \\
\hline $\begin{array}{l}\text { Moyenne : vache par jour } \\
\mathrm{E}=0,0768\end{array}$ & 2,96 & 3,18 & 2,99 & 0,435 \\
\hline
\end{tabular}

GROUPE DE VAGHES TEMMOIN :

VARIATION DU CONTENU EN PROTÉINES DU LAIT

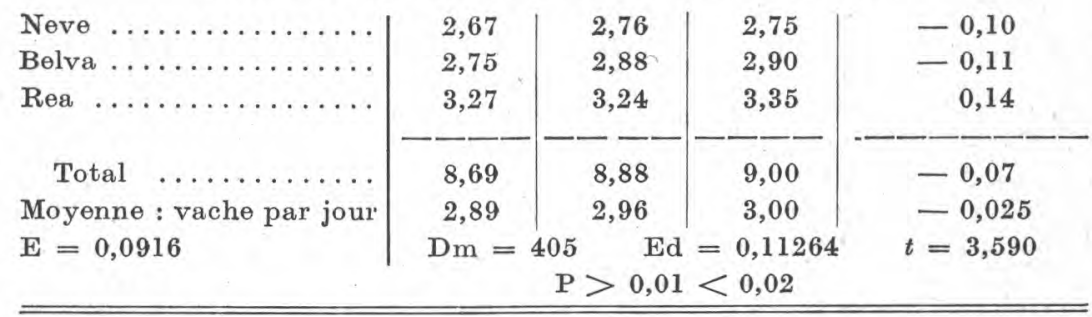

L'effet de l'administration du TM5, même si pour toutes les vaches des deux groupes le contenu en protéines penche vers l'augmentation, est particulièrement évident, ce que les résultats 
d'analyse statistique desquelles résulte une grande signification démontrent clairement.

\section{Deuxième expérience}

Matériel et technique. - Les recherches ont été conduites sur six vaches Brune Alpine du même âge, du même nombre de parts et de la même période de lactation.

On les tint dans les mêmes conditions d'ambiance et d'alimentation pendant toute la durée de l'expérience, et on leur administra pendant la deuxième période (de 30 jours) 1 gr. 5 de TM5.

La technique adoptée dans ces recherches a été la même que celle employée dans l'expérience précédente; n'ayant pu cependant disposer d'animaux témoins la comparaison fut effectuée entre les moyennes des productions de la deuxième période (celle du traitement antibiotique) et celles de la première et de la troisième; dans ce cas également, on réalisa l'élaboration des données suivant la méthode de BRANDT.

Résultats. - Dans les trois tableaux ei-après on a rapporté les valeurs moyennes des caractères examinés.

TABLEAU IV

EFFETS DE L'ADMINISTRATION DU TMS SUR LA PRODUGTION DU LAIT

\begin{tabular}{|c|c|c|c|}
\hline Nom de la vache & $\begin{array}{c}\text { Ire } \\
\text { période } \\
\text { Kg. }\end{array}$ & $\begin{array}{l}\text { II } \\
\text { période } \\
\text { Kg. }\end{array}$ & $\begin{array}{l}\text { III } \\
\text { période } \\
\text { Kg. }\end{array}$ \\
\hline Asia $\ldots \ldots \ldots \ldots \ldots$ & 13,00 & 15,67 & 14,05 \\
\hline 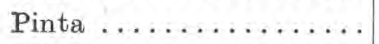 & 21,57 & 24,08 & 22,40 \\
\hline Oliva $\ldots \ldots \ldots \ldots \ldots$ & 22,66 & 25,88 & 23,39 \\
\hline Bologna ............. & 13,25 & 14,38 & 13,95 \\
\hline 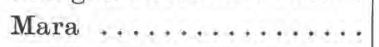 & 18,00 & 21,58 & 18,00 \\
\hline Amelia...$\ldots \ldots \ldots$ & 15,44 & 19,57 & 17,66 \\
\hline Total $\ldots \ldots \ldots \ldots$ & 103,920 & 121,160 & 109,450 \\
\hline Moyenne : vache par jour & 17,320 & 20,190 & 18,240 \\
\hline
\end{tabular}

Différences entre les moyennes
II $-I=2,87$
$t=7,172$
$\mathrm{Ed}=2,5145$
II - III $=1,95$
$t=8,830$
$\mathrm{P}<0,01$
$\mathrm{Ed}=2,7037$
$\mathrm{P}<0,01$

Considérant ces données, on voit que pendant la période de traitement la quantité de lait augmente, en comparaison avec les deux autres périodes, et que les effets du TM5 sont significatifs du point de vue statistique. 
TABLEAU V

EFFETS DE L'ADMINISTRATION DU TM5

SUR LE GONTENU EN MATIERE GRASSE DU LAIT

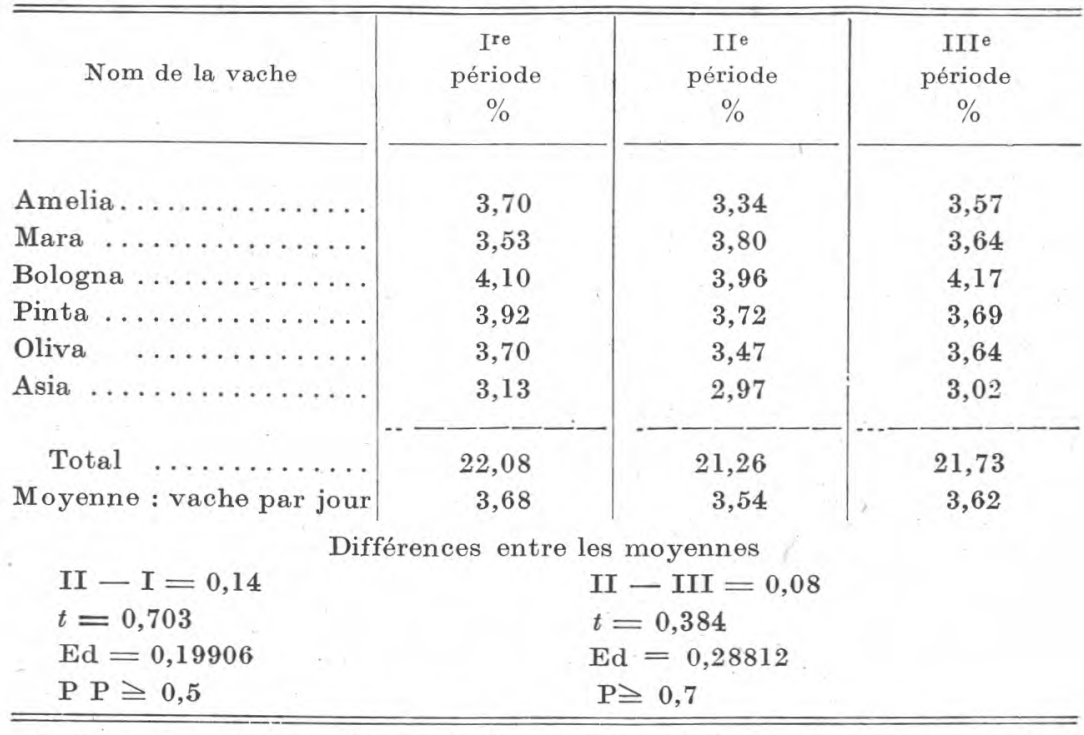

Comparant les valeurs de la période où les animaux recevaient le TM5 [11], avec celles de la première et de la troisième, on voit que l'administration cause une diminution; les différences cependant n'ont pas une valeur significative.

TABLEAU VI

EFFETS DE L'ADMINISTRATION DU TM5 SUR LE GONTENU EN PROTÉINES DU LAIT

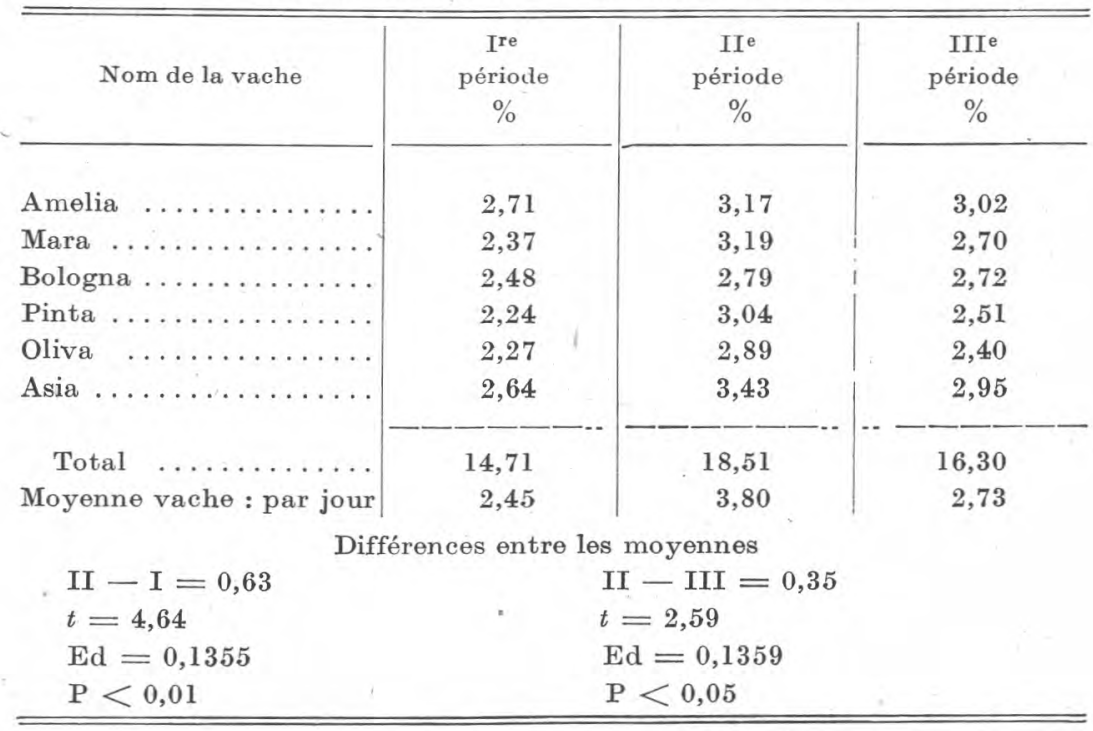


Considérant les valeurs rapportées, on voit que le contenu protéique du lait augmente à la suite de l'administration de l'antibiotique. L'analyse statistique met en évidence qu'il existe une grande signification seulement dans la comparaison entre les valeurs de la période de traitement et celles de la première période.

\section{Troisième expérience}

Matériel et technique. - Le but de la recherche a été de constater si l'administration, par voie orale, de petites quantités de terramycine cause des effets nuisibles sur la production du fromage. A cet effet, on a traité journellement et pendant 26 jours, 37 vaches avec 1 gr. 5 de TM5; le groupe témoin comprenait 79 vaches.

Les animaux du groupe expérimental et les témoins furent tenus, pendant toute la période de l'expérience, dans les mêmes conditions d'ambiance et d'alimentation.

Le lait de chaque groupe, journellement et séparément, fut employé pour la fabrication du fromage "Grana" (Parmesan), lequel, après le travail fut tenu pendant toute la période de maturité dans la même pièce. Les faits qui ont frappé davantage le fromager, pendant la fabrication du fromage avec le lait des vaches ayant reçu le TM5 sont : meilleur ouvrage, caillage plus consistant, moindre demande de sérum, temps de caillage et durée totale du travail plus faible, fromage plus doux.

Dans les contrôles périodiques effectués pendant la maturation, c'est-à-dire du 26 juillet 1957 au 20 décembre 1958, on ne remarqua aucune différence à l'égard de la diminution et des autres caractères macroscopiques des fromages.

Dans le tableau ci-après on a rapporté les valeurs trouvées à la suite des analyses exécutées, à la fin de la maturation, sur quelques spécimen.

TABLEAU VII

\begin{tabular}{|c|c|c|c|c|c|c|}
\hline \multirow{2}{*}{$\begin{array}{c}\text { Constituants } \\
\%\end{array}$} & \multicolumn{3}{|c|}{$\begin{array}{c}\text { Fromages } \\
\text { groupe expérimental }\end{array}$} & \multicolumn{3}{|c|}{$\begin{array}{l}\text { Fromages } \\
\text { groupe témoin }\end{array}$} \\
\hline & $\begin{array}{l}29-7-57 \\
\text { (1) }\end{array}$ & $\begin{array}{l}16-8-57 \\
(1)\end{array}$ & Moyenne & $\begin{array}{l}29-7.57 \\
\text { (1) }\end{array}$ & $\begin{array}{c}16 \cdot 8 \cdot 57 \\
\text { (1) }\end{array}$ & Moyenne \\
\hline Extrait sec ......... & 70,43 & 74,02 & - & 73,78 & 74,52 & - \\
\hline Protéines totales ...... & 49,62 & 46,86 & 48,24 & 47,14 & 50,00 & 48,57 \\
\hline Matière grasse $\ldots \ldots \ldots$ & 41,81 & 45,92 & 43,86 & 43,85 & 41,96 & 42,90 \\
\hline Cendres $\ldots \ldots \ldots \ldots \ldots$. & 5,73 & 5,91 & - & 6,03 & 6,49 & - \\
\hline Total $\ldots \ldots \ldots \ldots$ & $-\overline{97,16}$ & $-\cdot-$ & - - & $-\overline{97,02}$ & $\overline{98,45}$ & $-\cdots$ \\
\hline
\end{tabular}

(1) Date de fabrication des fromages. 
Comme on le remarque à l'examen du tableau, il n'y a pas de différence substantielle entre la composition chimique des fromages obtenus avec le lait des vaches traitées et ceux des vaches témoins.

D'après les résultats de nos expériences, il nous semble pourtant pouvoir conclure que l'administration journalière, par voie orale de 1 gr. 5 de TM5 cause chez les vaches en lactation une plus grande production de lait, une teneur plus élevée en protéines totales et une légère diminution de matière grasse.

On n'a constaté aucun effet nuisible ni sur la coagulation du lait ni sur la maturation du fromage.

Considérant les assertions du fromager, l'antibiotique aurait exercé une action favorable sur le temps de coagulation et sur la qualité du caillage.

\section{Summary}

The authors carried out three experiments on cows during the lactation period in order to ascertain the effects of terramycin (TM5) administered per os on the composition and quality of milk and on its cheese making properties.

In the light of the results obtained, they conclude that a significant increase is registered only in the production of milk and in total protein content. No harmful effect was noted in connection with cheese production and ripening.

\section{BIBLIOGRAPHIE}

[1] La Revue de l'Elevage, 1957, 1, 215.

[2] W. A. Krienne. Cité par Wilkowske et Krienke, voir [6].

[3] C. Arnaudi. Relazione al Congresso di Igiene di Napoli, 1956.

[4] C.J.E. Hunter. "The effect of penicillin on lactic streptococci." Cité par WILKOW SKE et KRIENKE, voir [6].

[5] R. E. Habgrove, H. E. Walter, J. P. Malkames, J. et K. T. Maskell. "The effect of penicillin and streptomycin on swiss cheese starter." J. Dairy Sci., 1959, 401.

[6] H. H. Wilkowske et W. A. KRIEnKe. "Influence of penicillin on the acid lactic production of certain in lactobacilli." J. Dairy Sci., 1951,1030 .

[7] H. H. Wilkowske et W. A. KrIEnke. "Assay of various mold-ripened cheeses for antibiotic activity. "J. Dairy Sci., 1954, 1184.

[8] R. G, Warner. Cité par LAssiter, voir [16].

[9] J. K. Loosil et R. G. WARner. Cités par Lassister, voir [16].

[10] E. E. Bartley, F. C. Fountaine et F. W. Amkeson. Antibiotic in dairy cattle nutrition. II. Effects of feeding an aureomycin product (Aureofac $2 \mathrm{~A}$ ) to lactating dairy cows. J. Dairy Sci., 1953, 402.

[11] W. H. Martin, T. J. Claydon et E. E. Bartley. "Aureomycin content, bacterial development, starter activity, and cheese quality of 
milk from cows fed an Aureomycin supplement. " J. Dairy Sci. $1955,47$.

[12] Huffman. Cité par Lassister, voir [16].

[13] M. O. HaQ, L. L. Rusoff. Cités par Lassister [16].

[14] M. O. Haq, L. L. Rusoff a A. J. Gelpi. Cités par Lassister [16].

[15] L. L. Rusoff a. M. O. HAQ. "Studies on aureomycin and Vit. B12 supplementation for dairy cows. II. Effect on production, composition and vitamin B12 content on the milk. » J. Dairy Sci. 1954, 677.

[16] C. A. LAssiter. "Antibiotic as growth stimulants for dairy cattle. A review. J. Dairy Sci. 1955.

\title{
AU SUJET DU VIRAGE DE LA RESAZURINE DANS LES LAITS FRAIS ADDITIONNÉS DE LAIT RECONSTITUÉ
}

\author{
par
}

VALENTIN TOUBOL

Directeur du Laboratoire Officiel d'Analyses et de Recherches Chimiques (Casablanca)

J'ai eu l'occasion, dans cette revue $(1956,86,385)$ de faire connaître mon point de vue au sujet des diverses méthodes que l'on trouve dans la littérature, concernant la détection du lait reconstitué dans le lait frais et toute l'importance que cette question représentait pour la recherche de cette fraude.

Le Docteur Intieri, poursuivant des recherches dans ce but (Il Latte - Milan, 1957) publiait récemment une réaction très simple dont la technique a été reprise et mise au point, pour en perfectionner la fidélité, par le Docteur Vétérinaire G. Belle, et M. P. Caspar, Directeur technique de la Centrale laitière de Casablanca (1).

Cette technique consiste à préparer dans du formol une solution de résazurine et d'en introduire dix gouttes dans dix centimètres cubes de lait étudié, après que ce lait ait subi un pré-chauffage à $37-40^{\circ}$ pendant cinq minutes.

Les colorations obtenues un quart d'heure après avoir ajouté le réactif varient $\mathrm{du}$ rose, pour le lait frais pur, au bleu pour le lait reconstitué à $100 \%$.

Notons tout d'abord que le lait reconstitué comme le lait cuit donnent des teintes analogues. Du point de vue de la fraude, l'addition de lait cuit, notamment pour des raisons d'hygiène, n'a pas le même caractère frauduleux que l'addition de lait en poudre généralement écrémé.

(1) Le Lait. 1959, 39, 241. 